\title{
De Quervain's Tenosynovitis: A Systematic and Citation Network Analysis Review
}

\author{
Tam Eunice Wai-si ${ }^{1}$, Yip Joanne ${ }^{1 *}$, Fang Christian ${ }^{2}$, Lo Kwan Yu ${ }^{1}$, Yick Kit Lun ${ }^{1}$ and Ng Sun Pui ${ }^{3}$ \\ ${ }^{1}$ Institute of Textiles and Clothing, The Hong Kong Polytechnic University, Hong Kong \\ ${ }^{2}$ Department of Surgery, University of Hong Kong Medical Center, Queen Mary Hospital \& Tung Wah Hospital, Sheung Wan, Hong Kong \\ ${ }^{3}$ Division of Science, Engineering and Health Studies. Hong Kong Community College, Hong Kong
}

*Corresponding author: Yip Joanne, Institute of Textiles and Clothing, The Hong Kong Polytechnic University, Hong Kong

\begin{tabular}{l}
\hline ARTICLE INFO \\
\hline Received: 㓞 January 16, 2020 \\
Published: January 24, 2020 \\
\hline Citation: Tam Eunice Wai-si, Yip Joanne, \\
Fang Christian, Lo Kwan Yu, Yick Kit Lun, \\
Ng Sun Pui. De Quervain's Tenosynovitis: A \\
Systematic and Citation Network Analysis \\
Review. Biomed J Sci \& Tech Res 24(5)- \\
2020. BJSTR. MS.ID.004125.
\end{tabular}

Keywords: De Quervain's tenosynovitis; systematic review; citation network analysis; clustering; Markov Cluster Algorithm

Abbreviations: APL: Abductor Pollicis Longus; EPB: Extensor Pollicis Brevis; FDC: First Dorsal Compartment; WHAT: Wrist Hyperflexion and Abduction of the Thumb; CAN: Citation Network Analysis; RCTs: Randomized Controlled Trials; MCL: Markov Cluster Algorithm

\section{ABSTRACT}

\section{Study Design: Systematic review}

Introduction: De Quervain's tenosynovitis (DQV) is a common hand disorder causing pain along the radial side of the wrist in patients. Patients are mainly in the age groups of forties to sixties. They may feel difficulties in performing daily activities that involve the movement of thumb and wrist.

Purpose of the study: The objectives of the study is to conduct classification of articles that are related to DQV, to examine the publication trend and main research areas of this hand condition and to indicate the research opportunities for future studies.

Methods: Articles related to DQV dated between 1970 to Feb 2019 were searched on the Web of Science database. Searching keywords include derivations and general terms of 'DQV'.

Results: 197 articles that met the inclusion criteria were identified and evaluated by the citation network analysis. Eleven main clusters were classified.

Discussion: The two dominant clusters are 'Effectiveness and Comparisons of Treatments and Combined Therapies' and 'Ultrasound-guided Injection and Injection Accuracy'. Other main clusters focus on anatomical studies of the first dorsal compartment, alternative approaches to surgery for complications minimization, epidemiological studies, studies on specific types of patients, radiographic evaluation, and diagnostic tools of de Quervain's tenosynovitis. Based on the clustering results, future research directions for studies on de Quervain's tenosynovitis are provided accordingly.

Conclusion: Articles related to DQV mainly compare and focus on the effectiveness of treatments. Studies on orthotic treatments are limited that further research on this area should be conducted. More studies exploring the hand conditions in younger age groups, pregnant and postpartum women with DQV are also necessary (Graphical Abstract 1).

\section{Introduction}

De Quervain's tenosynovitis (DQV) is a painful condition in which patients may feel tenderness and soreness at the radial side of the wrist near the radial styloid [1]. Therefore, daily activities that involve wrist and thumb movements may be affected [2]. Patients may find it difficult to perform tasks that require turning of the wrist and exerting force by the thumb, such as opening jars with lids, lifting a child, etc. [3].

DQV is an inflammation condition of the synovial sheath that surrounds the abductor pollicis longus (APL) and the extensor pollicis brevis (EPB) tendons of the first dorsal compartment (FDC). 
The occurrence of neutrophil elastase and cyclooxygenase, and the corresponding effects on the collagen structure in the extensor retinaculum samples collected from patients with $D Q V$, indicate the presence of inflammation in the FDC in DQV [4].

However, some studies have mentioned that despite the term 'tenosynovitis' in DQV, there is actually no inflammation involved.
The thickening of the synovial sheath is due to degenerative changes, such as myxoid degeneration [5,6] and may cause the tightening of the tendon canal during movement of the APL and EPB tendons $[3,7]$. Thus, there is greater friction between the two tendons and the tendon canal, which could produce pain and tenderness over the FDC [1].

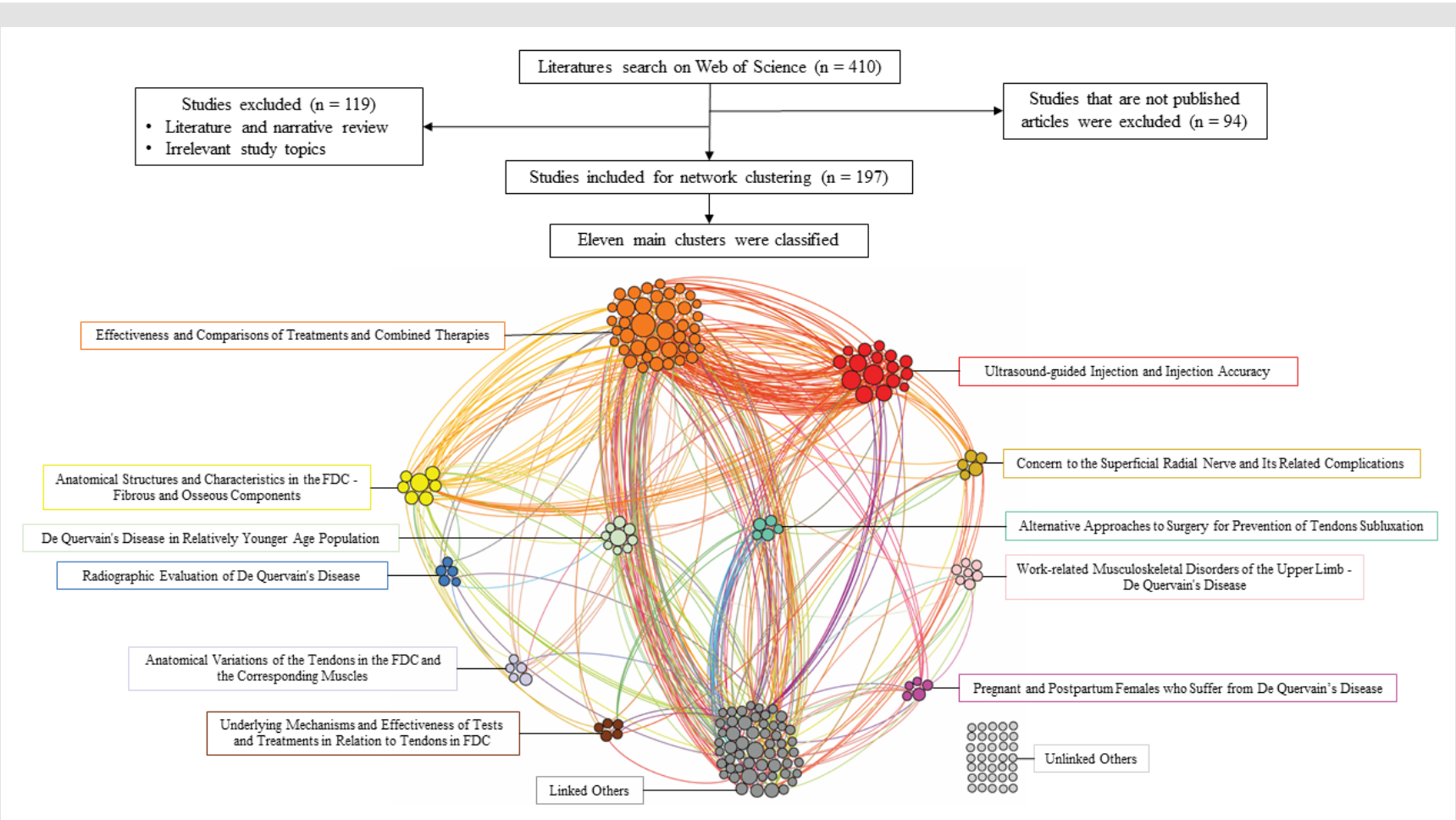

Graphical Abstract 1.

The prevalence of DQV is $0.5 \%$ in men and $1.3 \%$ in women among the general population [5], so women will have DQV more than men [8]. Patients are usually in their forties to sixties [9]. Risk factors include the overuse of the hands, and repetitive hand activities that depend on wrist and thumb movements [10]. Le Manac'h, et al. [11] carried out a study that assessed the occupational risk factors of DQV in French workers such as working tasks that required sustained periods of wrist bending or extreme wrist postures, and repetitive hand movements like driving screws. Those who are employed in occupations that require repetitive and forceful hand movements, such as musicians and machinists, face an increased risk of DQV. Postpartum women are also at risk of DQV as they are constantly lifting their babies [10]. A number of studies have shown that anatomical variations in the FDC, such as the presence of septum and multiple slips of the tendon are associated with DQV [12,13].

The diagnostic test for DQV is the Finkelstein's test, in which patients are required to flex their thumb and enclose their thumb with a closed fist. While holding this specific hand posture, ulnar deviation of the wrist is either actively carried out by patients or passively by the clinician. Pain experienced at the radial side of the wrist near the radial styloid process is a positive indicator of DQV
[3]. Other tests have also been proposed, such as Brunelli's test and the Wrist Hyperflexion and Abduction of the Thumb (WHAT) test $[1,14]$. For mild and acute cases, conservative treatments include orthotic intervention, rest, taking anti-inflammatory medication, applying heat and cold, massage and exercises [2,7,10]. A study revealed that the symptoms of mild DQV in 15 out of 17 patients are completely alleviated through a combination of orthotic intervention and oral intake of non-steroidal anti-inflammatory drugs [15].

For patients who are experiencing moderate wrist pain, corticosteroid injections may be advised. A retrospective study by Hajder, et al. [5] found that the injections of triamcinolone with an ultrasound-guided technique is safe and effective for $91 \%$ of the patients. Surgery may also be prescribed for those who cannot find relief with conservative treatments. Ta, et al. [16] found that $91 \%$ of the patients in their study experienced no complications after surgery. Previous studies have mainly focused on treatment. For example, Ashraf, et al. [17] conducted a systematic review and meta-analysis to determine the effectiveness of steroid injection as a treatment in comparison to orthotic intervention in adults. Cavaleri, et al. [18] included 6 eligible studies for a systematic review and meta-analysis, with the aim to examine the effectiveness 
of different therapeutic approaches and compare the differences in therapies, for example, orthosis treatment versus corticosteroid injections. They concluded that combined therapies of orthosis use and corticosteroid injection are more effective than any of the standalone treatments. Huisstede, et al. [19] conducted an evidencebased systematic review on the effectiveness of conservative treatment, surgical and post-surgical interventions for three hand disorders, including DQV. Besides, Stahl, et al. [20] conducted a meta-analysis on investigating the relationship between DQV and work-related manual tasks, and further examined the causal relationship between occupational risk factors and DQV. Although systematic reviews related to DQV are available, the majority have focused on a specific area of DQV. Furthermore, related systematic reviews are still minimal. Instead, research related to DQV covers broad areas, such as anatomy, diagnostic tests and treatments. This paper therefore aims to provide a comprehensive and systematic review on DQV with the following objectives:

Table 1: Keywords. a. To classify the articles related to DQV by year of publication, country of origin of authors, journal name, study design and methodology,

b. To determine the main research domains of DQV with a citation network analysis and

c. To indicate the research trend and research opportunities for future studies on DQV

\section{Methodology}

A literature search was conducted by using the advanced search on the Web of Science database that involved two sets of keywords. The first set of keywords is a list of derivations of 'DQV', and the second set of keywords is a list of other names that refer to 'DQV' and often used by the general public; see Table 1 . The review period is from 1970 to Feb 2019. The following are the exclusion criteria:

\begin{tabular}{|c|c|}
\hline Root Term & De Quervain's Tenosynovitis \\
\hline Search terms 1 & $\begin{array}{l}\text { (Quervain OR “Quervain's" OR “de Quervain" OR “de Quervain's" OR deQuervain OR “deQuervain's") AND (tenosynovitis } \\
\text { OR synovitis OR tenovaginitis OR tendovaginitis OR tendinitis OR tendonitis OR tendinosis OR tendinopathy OR peritendinitis } \\
\text { OR paratenonitis OR syndrome OR disease) }\end{array}$ \\
\hline Search terms 2 & "Blackberry thumb" OR “Gamer's thumb" OR “Washerwoman thumb" OR "Mother's thumb" OR "Mummy thumb" \\
\hline
\end{tabular}

a. Other types of documents, such as proceeding papers, letters, etc., and

b. Studies not related to DQV that focus on other disease, for example de Quervain's thyroiditis and other hand disorders that are mimicking DQV like osteoid osteoma of the radial styloid and brachial artery thrombosis.

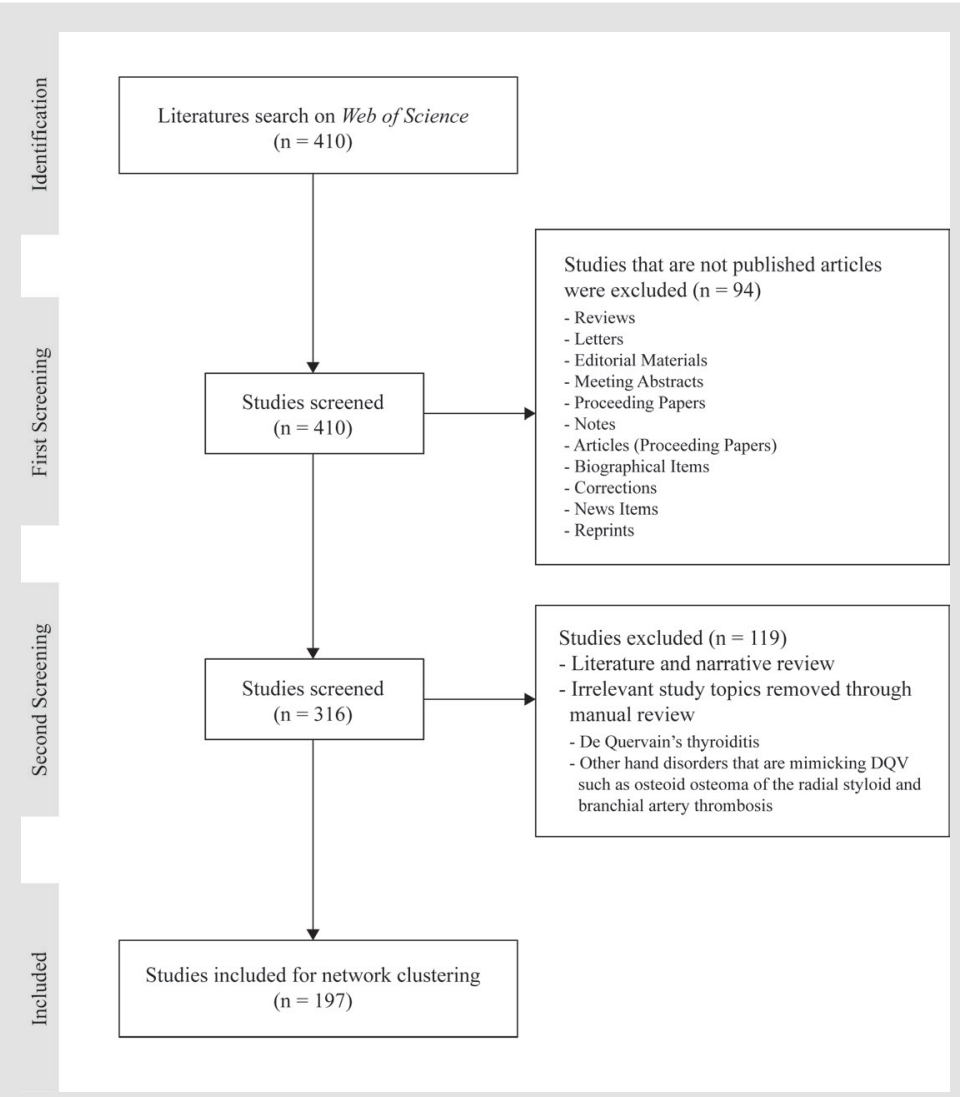

Figure 1: Flow chart of selection process. 
Articles included are in English. The number of studies found in the literature is 410 . The list was downloaded and imported into Histcite software to organize and analyze the retrieved papers [21] and identify and exclude papers other than journal articles. A manual review of the title and abstract of each paper excludes any remaining irrelevant papers. Finally, 197 articles were shortlisted to create the citation network (Figure 1).

The shortlisted articles were subjected to a citation network analysis (CNA) which offers an objective analysis by visualizing the interconnectivity of researchers, methodologies or articles based on their citation records $[21,22]$. The analysis can identify and then sort studies into different clusters or subgroups of studies that belong to the same or similar research areas which tend to review and cite each other. Furthermore, some other studies may be identified as dissimilar and isolated [22]. The CNA presents a list of the literature in the form of a network with nodes that represents a single study and links that represent the citation connections between the studies. In a directional CNA, arrowheads appear at the links, which represents the 'cite' and 'cited' relationships between studies and depicts the flow of knowledge [21,23]. Histcite, which can produce a citation index for studies retrieved from the ISI Web of Science [24], was used to export the study information and the corresponding citation data into a Pajek file, which was then imported into Gephi software to plot the graphs and visualize the networks [25].

\section{Results}

\section{Descriptive Statistics}

In this section, the classification of the shortlisted articles is discussed in detail. Articles with an experimental design were further categorized based on the different methodologies. An overview of the research trend in DQV and study distribution was obtained from the descriptive statistics.

\section{Year of Publication}

The plotted distribution of articles related to DQV is shown in Figure 2. There is a gradually growing trend of published articles related to DQV. The total number of recently published articles from 2013 to 2018 is 89 (approximately 45.18\%). Thus, most of the articles have been published just recently.

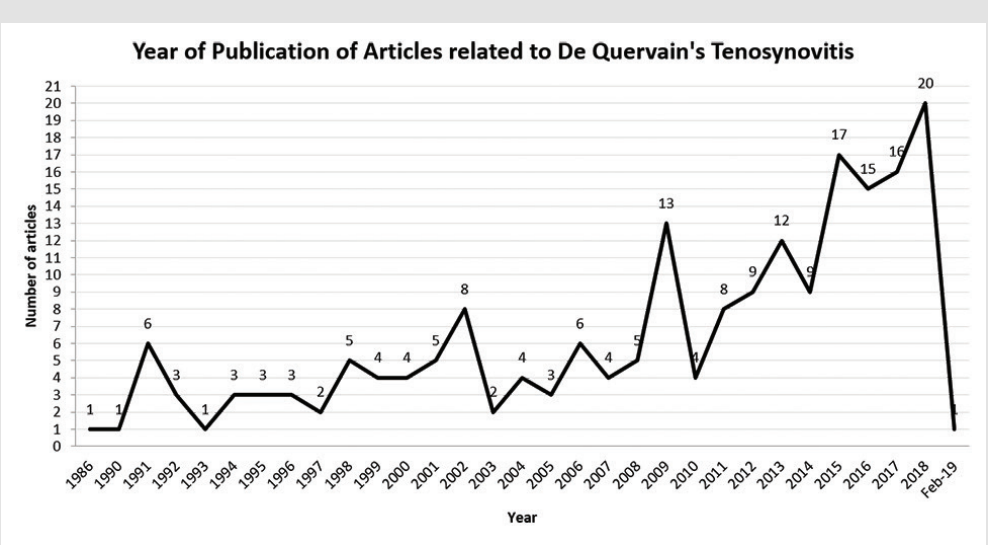

Figure 2: Article distribution by year of publication

\section{By Country}

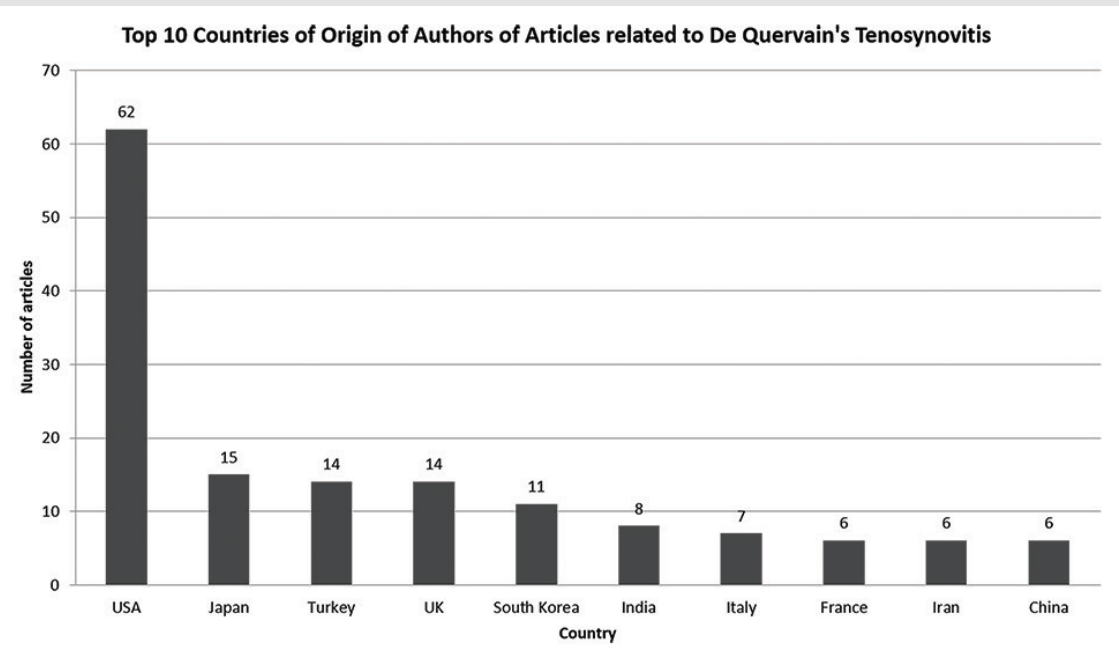

Figure 3: Article distribution by country. 
The articles were also classified and then plotted by the country of origin of the authors, based on the provided address of the article. Thus, this distribution could point to where the studies described in the articles were actually carried out. Figure 3 shows the top 10 countries. Sixty-two or $31.47 \%$ of the articles are published by individuals in the USA. There are comparatively fewer articles from the other top nine countries, with only approximately half of the number of articles or less. Fifteen articles (7.61\%) are written by those in Japan, 14 articles (7.11\%) in Turkey and the UK respectively, and 11 articles (5.58\%) in South Korea. As for the remaining five countries, less than 10 articles were published, which included India with 8 articles (4.06\%), Italy with 7 articles (3.55\%), and France, Iran and China with 6 articles (3.05\%) respectively.

\section{Article Distribution by Journal Name}

Figure 4 shows the top five journals that have published on DQV. Twenty-five articles are published by the Journal of Hand Surgery - American Volume, or $12.69 \%$. The number of articles published in four other journals is half or less than half of that published in the leading journal. Ten articles (5.08\%) are found in the
Journal of Hand Surgery - European Volume and 7 articles (3.55\%) in the Journal of Hand Surgery - British and European Volume. Both the American Journal of Industrial Medicine and International Orthopaedics have published 6 related articles (3.05\%). The three journals with the most publications on DQV are all Journal of Hand Surgery based in different countries. According to the database in the InCites Journal Citation Reports, they focus on orthopedics and surgery. Similarly, International Orthopaedics also publishes mainly on orthopedics. The American Journal of Industrial Medicine focuses on public, environmental and occupational health. Therefore, articles related to DQV are mainly published in journals that focus on orthopedics, surgery and health.

\section{Study Design}

Figure 5 shows the distribution of the articles based on their study design. In total, 112 articles in the sample (56.85\%) are observational studies, or more than half of the shortlisted articles, 49 are experimental studies and 33 are case reports. The latter two account for $24.87 \%$ and $16.75 \%$ of the articles, respectively. Finally, only 3 articles are conceptual studies.

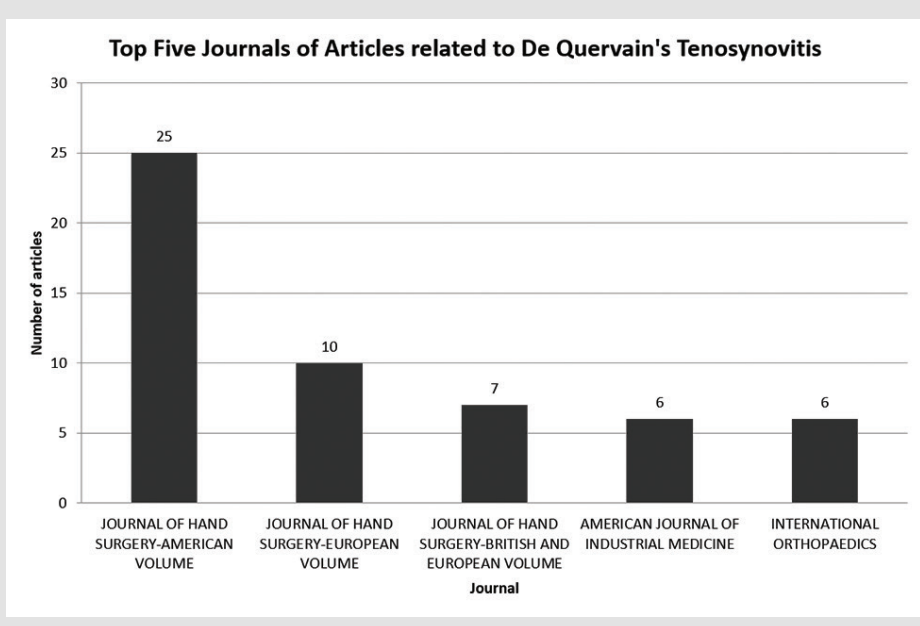

Figure 4: Article distribution by journal.

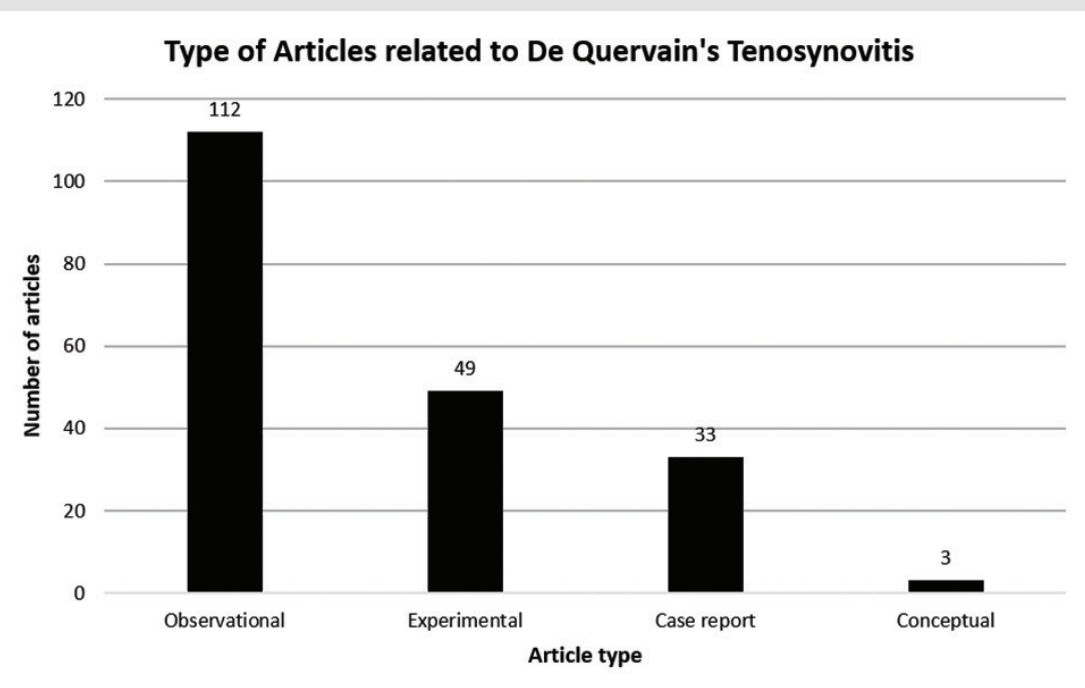

Figure 5: Article distribution by study design. 


\section{Methodology in Experimental Studies}

According to Song, et al. [26], experimental studies can be subdivided into uncontrolled trials and controlled trials. The former are trials without a control group, while the latter include a control group for comparison purposes. They also stated that controlled trials can be further subdivided into non-randomized trials, trials without randomized allocation of subjects into intervention and control groups, and randomized controlled trials (RCTs) with subjects randomly allocated. In this study, the articles with an experimental study design are further categorized into uncontrolled trials, non-randomized controlled trials and RCTs, as shown in Figure 6. There are 21 articles (42.86\%) with uncontrolled trials and 28 articles (57.14\%) with controlled trials. In the latter, 8 articles (28.57\%) are non-randomized controlled trials and 20 (71.43\%) are RCTs.

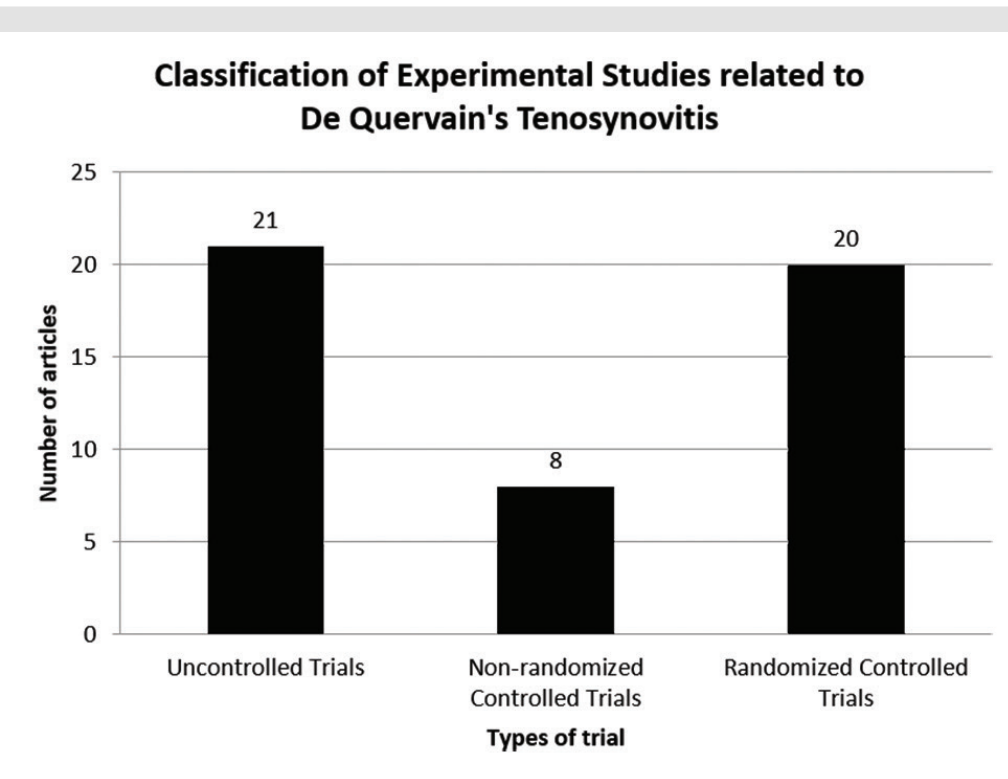

Figure 6: Article distribution by study design.

\section{Clustering of Articles}

The Markov Cluster Algorithm (MCL), a clustering algorithm developed by Van Dongen [27], is well known for its effectiveness in graph clustering, reliability and robustness and therefore adopted here [28]. The MCL involves two steps to carry out random walks on a graph that show gathering of flow, which are 'expansion' and 'inflation'. The algorithm assumes that a random walk from node to node will tend to remain within a connected and more linked cluster, with a low probability of crossing the boundaries of another cluster, in which the resulting clustering is based on the existing network structure [29]. In this study, the linkages between nodes are the citation relationships with each node representing an article, which comprise the citation network structure. As the literature was objectively clustered by using the MCL, the modularity value $Q$ which measures the strength of a citation network structure [25], was taken into consideration. The following shows the equation for Q

$$
Q=\sum_{s=1}^{k}\left[\frac{m_{s}}{m}-\left(\frac{d_{s}}{2 m}\right)^{2}\right]
$$

where $S$ represents the cluster with $k$ that indicates the number of clusters. $m_{s}$ and $d_{s}$ are respectively the total number of edges and the sum of the degree of nodes in cluster $\mathrm{S}$. The total number of edges is indicated as $\mathrm{m}$ [30]. $\mathrm{Q}$ is an index for the evaluation of clustering accuracy, which normally falls in the range from 0.3 to 0.7 [31].

The citation network of studies related to DQV is developed as shown in Figure 7. Q is 0.415 , which fell into the mentioned normal range. The nodes are illustrated in different sizes based on their degree. The degree of a node denotes the number of nodes that is connected to this particular node, which is the number of inward and outward connections to and from a node in a directed graph [25]. The clustering of the 197 articles in the sample by using the MCL was processed in Gephi, which resulted in 67 clusters. Of those, 56 clusters were defined as scattered groups with less than 5 articles each. Within these 56 clusters, 21 clusters (dark grey nodes) with 54 articles are scattered groups with citation connectivity to the main clusters (27.41\%), while 35 clusters (light grey) with one article are scattered articles without connections to the main clusters $(17.77 \%)$. Eleven main clusters with 5 or more articles in each cluster are identified. The most popular research area in DQV focuses on the effectiveness and comparisons of treatments and combined therapies for DQV (orange), which comprise 39 articles (19.80\%). The second most popular research area is also about the treatment of DQV, mainly focusing on ultrasound-guided injection and the injection accuracy (red), with 16 articles (8.12\%). Nine articles $(4.57 \%)$ focus on the relatively younger population (light green). 
The number of articles on work-related musculoskeletal disorders of the upper limbs which includes DQV (pink) and anatomical structures and characteristics of the fibrous and osseous components of the FDC (yellow) is 7 (3.55\%) for both areas, followed by 5 articles $(2.54 \%)$ on 6 other areas respectively, which are the anatomical variations of the tendons in the FDC and the corresponding muscles (light purple), DQV in pregnant and postpartum females (reddish purple), alternative approaches to surgery (green), superficial radial nerve and its related complications (ochre), radiographic evaluations (blue), and the underlying mechanisms and effectiveness of tests and treatments in relation to tendons in FDC (brown). Figure 7 shows the citation network. Table 2 shows the cluster theme based on the cluster number. Finally, Figure 8 shows the number of articles in each cluster.

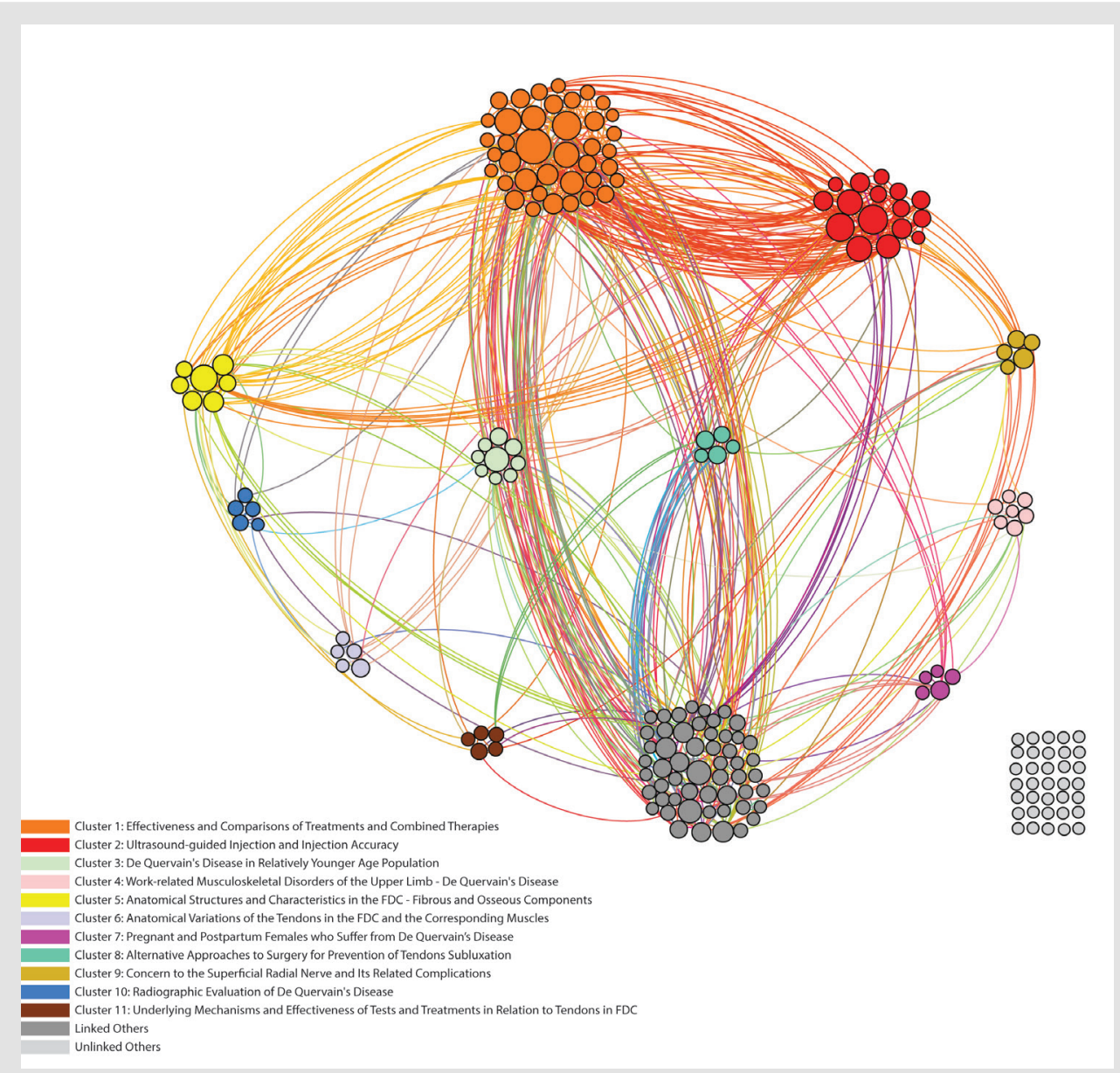

Figure 7: Citation network of articles related to DQV.

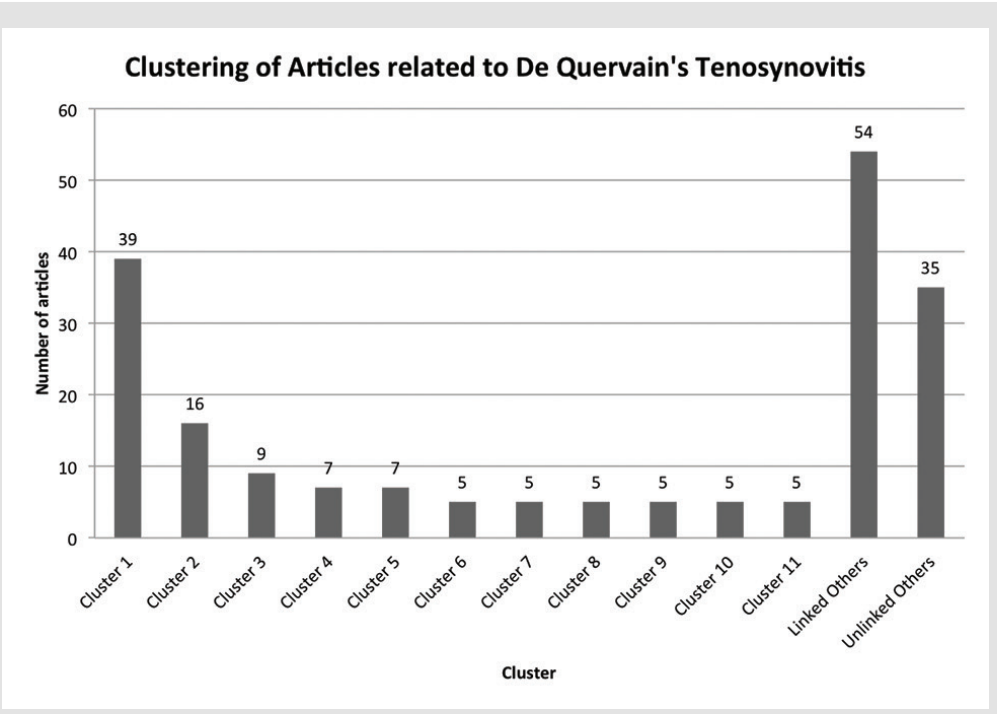

Figure 8: Clustering of sample articles. 
Table 2: Cluster themes.

\begin{tabular}{|c|c|}
\hline \multicolumn{2}{|r|}{ Cluster Themes } \\
\hline Cluster 1 & $\begin{array}{c}\text { Effectiveness and Comparisons of Treatments and } \\
\text { Combined Therapies }\end{array}$ \\
\hline Cluster 2 & Ultrasound-guided Injection and Injection Accuracy \\
\hline Cluster 3 & De Quervain's Disease in Relatively Younger Age Population \\
\hline Cluster 4 & $\begin{array}{c}\text { Work-related Musculoskeletal Disorders of Upper Limbs - } \\
\text { De Quervain's Disease }\end{array}$ \\
\hline Cluster 5 & $\begin{array}{l}\text { Anatomical Structures and Characteristics in FDC - Fibrous } \\
\text { and Osseous Components }\end{array}$ \\
\hline Cluster 6 & $\begin{array}{c}\text { Anatomical Variations of the Tendons in the FDC and } \\
\text { Corresponding Muscles }\end{array}$ \\
\hline Cluster 7 & $\begin{array}{l}\text { Pregnant and Postpartum Females who Suffer from De } \\
\text { Quervain's Disease }\end{array}$ \\
\hline Cluster 8 & $\begin{array}{l}\text { Alternative Approaches to Surgery for Prevention of Tendon } \\
\text { Subluxation }\end{array}$ \\
\hline Cluster 9 & $\begin{array}{l}\text { Concern to the Superficial Radial Nerve and Its Related } \\
\text { Complications }\end{array}$ \\
\hline Cluster 10 & Radiographic Evaluation of De Quervain's Disease \\
\hline Cluster 11 & $\begin{array}{l}\text { Underlying Mechanisms and Effectiveness of Tests and } \\
\text { Treatments in Relation to Tendons in FDC }\end{array}$ \\
\hline
\end{tabular}

\section{Discussion}

As DQV is one of the common musculoskeletal disorders of the upper limbs, the distribution of articles by publication year in this study also shows a growing trend of research on DQV. Observational studies are the most prevalent, which mainly include anatomical studies of the FDC, imaging and effectiveness of radiographic tools, evaluation of diagnostic tests, and survey studies on upperlimb disorders. The latter provide the rate of prevalence of DQV in different countries, regions and industries, for example Mexico [32], Latin-America [33] and Colombia [34]. In comparison to observational studies, the number of experimental studies on DQV is comparatively less. Experimental studies are important research on the efficacy and effectiveness of different types of treatments for DQV. The results may have impacts on managing treatment for DQV patients. They may also contribute to the development of managing treatment for patients with different severity of DQV. Therefore, more experimental studies on DQV is necessary for the future development of the management of DQV.

Some studies are in the form of case reports, which may discuss the experiences of patients with DQV, or some rare, but special and remarkable cases that cover different aspects related to DQV, for instance, the suffering experience with DQV of a female nurse [35], patients with lymphedema who suffer from DQV [36], and rare complications after corticosteroid injection [37]. These case reports show that DQV is not a simple musculoskeletal hand disorder with clearly defined etiology and pathology, but a complicated condition with controversial causing factors, unexpected complications after treatment and potential linkage with other diseases. More studies may be needed to provide a more comprehensive picture of DQV.

The plotting of citation networks provides a clear graphical visual to show the citation connectivity between articles. Articles form clusters with those that have a stronger citation relationship. In this study, the two largest clusters in the citation network mainly focus on treatments with the majority of them being experimental studies although the number of observational studies exceeds that of experimental studies. This clustering result shows that there is more citation bonding between the experimental studies, in which researchers who examine similar types of treatment tend to cite articles with similar topics and background to support their experimental designs and add to their study contents and discussion. Furthermore, as observational studies cover different areas, such as anatomical observations, epidemiological studies and diagnostic tool evaluation, this may result in weaker citation connectivity between them.

The largest cluster includes articles that mainly focus on comparing the effectiveness between different types of treatments and combined therapies. Most of these studies involved corticosteroid injection as a treatment in their comparisons, for example, corticosteroid injection versus combined corticosteroid injection and orthotic treatment [38], corticosteroid injection and orthotic treatment versus orthotic treatment alone [39], and four-point versus two-point steroid injection [40]. Articles that fall into the second largest cluster are alternative approaches to conventional corticosteroid injection, like ultrasound-guided injection, and their effectiveness. Apart from research on injection, some researchers have focused on surgical treatment. They examine the long-term results of traditional surgical treatment or alternative approaches to surgery to minimize the emergence of post-surgical tendon subluxation and other complications. Citation relationships are found between studies on surgery, which result in two clusters called 'Alternative Approaches to Surgery for Prevention of Tendon Subluxation' and 'Concern to the superficial radial nerve and its related complications'. In comparing the number and citation networks of studies on injection and surgery, studies focus on orthotic intervention or other types of treatments are rarer with weaker citation connectivity, thus resulting in difficulties in forming specific clusters. Only two conceptual studies propose novel designs of orthoses for treatment of DQV, one experimental study compared the effectiveness of two orthoses with different designs, and a few studies examined other types of treatments, such as acupuncture, and ultrasonic and low-level laser therapies.

In the past few decades, more researchers have shown interest in the anatomical variations in the FDC. According to the clustering result here, some of them have mainly focused on studying the anatomical variations of the APL and EPB tendons and corresponding muscles, while some have investigated the fibrous and osseous components. Nayak, et al. [41] presented the case of a 51-year-old male cadaver with the complete absence of the EPB tendon and six APL tendon slips inserted into his forearm. Roy, et al. [42] highlighted the case where there is the presence of the bitendinous insertion of APL and accessory muscle in the left arm of an adult cadaver. Gurses, et al. [43] studied the wrists of 26 
cadavers to find the presence of septum in the FDC and examined the various types of radial groove. These researchers, who have contributed to the publications in the two anatomy-related clusters, demonstrate their interest and concern on the anatomical variation in FDC. This may also show that anatomical variations in FDC is associated with the occurrence of DQV.

DQV is also called 'Mother's thumb' or 'Mummy thumb' [18]. Thus, pregnant and postpartum women are potential sufferers of this hand disorder. The risk factors might be the changes in level of estrogen, progesterone and relaxin during pregnancy or the repetitive wrist and thumb related activities done during the caring of newborn babies [44]. The cluster 'Pregnant and Postpartum Females who Suffer from De Quervain's Disease' shows the group of researchers who have investigated DQV with this specific group of females as target subjects, in terms of prevalence rate and risk factors [45], anatomical structure of the FDC [46] and appropriate interventions [47]. Another cluster, which are studies that focus on DQV in relatively younger populations is also worthwhile of discussion. Turkay, et al. [48] examined the APL and EPB tendons of the FDC in $40 \mathrm{DQV}$ patients and 40 healthy participants with shear wave elastography. The median age of the DQV and healthy groups was 34 and 37.5 years-old respectively. Sreejith, et al. [49] compared the outcomes between conservative treatments and injection treatments in 92 patients and noted more patients in their thirties and forties. Rossi, et al. [50] examined DQV in volleyball players and the mean age is 24 years old. The studies in this cluster show that researchers are starting to see DQV patients who are under 40 years old. It is also evident that DQV studies of younger individuals have been increasing in number from 2013 to 2018.

\section{Limitations}

There are some limitations in this systematic study. First, there is 'selection bias'. Even though the first screening process is objectively carried out by using Histcite, the second screening process is partially subjective. Secondly, although the size of the node that represents the degree of connectivity of an article with cited works is shown in the citation network, the MCL algorithm may not include the weight and degree of connectivity of the article during the creation of the citation network. Therefore, future works would want to consider these two factors during algorithm calculation.

\section{Conclusion}

A systematic review of articles related to DQV is presented here, and distributions of the articles have been examined to gain a better understanding of articles on DQV in different areas. A citation network is created to identify the main clusters of research studies. The two dominant clusters are 'Effectiveness and Comparisons of Treatments and Combined Therapies' and 'Ultrasound-guided Injection and Injection Accuracy'. The number of studies related to DQV has been increasing. Since experimental studies on DQV only contribute to one-fifth of the entire sample here, more experimental studies should be carried out on the effectiveness of the different types of interventions and novel therapeutic modalities. As studies on orthotic treatment and alternative interventions are also limited, more research is important. Future research could also further explore pregnant and postpartum women who are with DQV. Furthermore, the younger age group with DQV could be a research target in future studies.

\section{Acknowledgement}

The work is supported by a research studentship granted to Ms. Tam Eunice Wai-si from The Hong Kong Polytechnic University.

\section{References}

1. Goubau JF, Goubau L, Van Tongel A, Van Hoonacker P, Kerckhove D, et al. (2014) The wrist hyperflexion and abduction of the thumb (WHAT) test: a more specific and sensitive test to diagnose de Quervain tenosynovitis than the Eichhoff's test. J Hand Surg Eur 39(3): 286-292.

2. Patel KR, Tadisina KK, Gonzalez MH (2013) De Quervain's disease. Eplasty 13: Ic52.

3. Goel R, Abzug JM (2015) De Quervain's tenosynovitis: a review of the rehabilitative options. Hand 10(1): 1-5.

4. Kuo YL, Hsu CC, Kuo LC, Wu PT, Shao CJ, et al. (2015) Inflammation is present in de Quervain disease-correlation study between biochemical and histopathological evaluation. Ann Plast Surg 74: S146-S151.

5. Hajder E, De Jonge MC, Van Der Horst CMAM, Obdeijn MC (2013) The role of ultrasound-guided triamcinolone injection in the treatment of de Quervain's disease: treatment and a diagnostic tool? Chir de la main 32(6): 403-407.

6. Read HS, Hooper G, Davie R (2000) Histological appearances in postpartum de Quervain's disease. J Hand Surg - Br \& Eur 25(1): 70-72.

7. Nemati Z, Javanshir MA, Saeedi H, Farmani F, Aghajani Fesharaki S (2017) The effect of new dynamic splint in pinch strength in de Quervain syndrome: a comparative study. Disabil Rehabil Assist Technol 12(5): 457-461.

8. Wolf JM, Sturdivant RX, Owens BD (2009) Incidence of de Quervain's tenosynovitis in a young, active population. J Hand Surg 34(1): 112-115.

9. Richie CA, Briner WW (2003) Corticosteroid injection for treatment of de Quervain's tenosynovitis: a pooled quantitative literature evaluation. J Am Board Fam Pract 16(2): 102-106.

10. Howell ER (2012) Conservative care of de Quervain's tenosynovitis/ tendinopathy in a warehouse worker and recreational cyclist: A case report. J Can Chiropr Assoc 56(2): 121-127.

11. Le Manach AP, Roquelaure Y, Ha C, Bodin J, Meyer G, et al. (2011) Risk factors for de Quervain's disease in a French working population. Scand J Work Environ Health 37(5): 394-401.

12. Kay NRM (2000) De Quervain's disease: changing pathology or changing perception? J Hand Surg 25(1): 65-69.

13. Minamikawa Y, Peimer CA, Cox WL, Sherwin FS (1991) De Quervain's syndrome: surgical and anatomical studies of the fibroosseous canal. Orthop 14(5): 545-549.

14. Brunelli G (2003) Finkelstein's versus Brunelli's test in de Quervain tenosynovitis. Chir de la main 22(1): 43-45.

15. Lane LB, Boretz RS, Stuchin SA (2001) Treatment of de Quervain's disease: role of conservative management. J Hand Surg Br \& Eur 26(3): 258-260.

16. Ta KT, Eidelman D, Thomson JG (1999) Patient satisfaction and outcomes of surgery for de Quervain's tenosynovitis. J Hand Surg 24(5): 10711077. 
17. Ashraf MO, Devadoss VG (2014) Systematic review and meta-analysis on steroid injection therapy for de Quervain's tenosynovitis in adults. Eur Orthop Surg \& Traumatol 24(2): 149-157.

18. Cavaleri R, Schabrun SM, Te M, Chipchase LS (2016) Hand therapy versus corticosteroid injections in the treatment of de Quervain's disease: A systematic review and meta-analysis. J Hand Ther 29(1): 3-11.

19. Huisstede BM, van Middelkoop M, Randsdorp MS, Glerum S, Koes BW (2010) Effectiveness of interventions of specific complaints of the arm, neck, and/or shoulder: 3 musculoskeletal disorders of the hand. An update. Arch Phys Med Rehabil 91(2): 298-314.

20. Stahl S, Vida D, Meisner C, Lotter 0, Rothenberger J, et al. (2013) Systematic review and meta-analysis on the work-related cause of de Quervain tenosynovitis: a critical appraisal of its recognition as an occupational disease. Plast Reconstr Surg 132(6): 1479-1491.

21. Colicchia C, Creazza A, Strozzi F (2018) Citation network analysis for supporting continuous improvement in Higher Education. Stud High Educ 43(9): 1637-1653.

22. Dorsch TE, Vierimaa M, Plucinik JM (2019) A citation network analysis of research on parent- child interactions in youth sport. Sport Exerc Perform Psychol 8(2): 145-162

23. Hummon NP, Dereian P (1989) Connectivity in a citation network: the development of DNA theory. Soc netw 11(1): 39-63.

24. Lucio Arias D, Leydesdorff L (2008) Main-path analysis and pathdependent transitions in HistCite ${ }^{\mathrm{TM}}$-based historiograms. J Am Soc Inf Sci Tec 59(12): 1948-1962.

25. Khokhar D (2015) Gephi Cookbook. Packt Publishing, Birmingham, UK. pp. 296.

26. Song JW, Chung KC (2010) Observational studies: cohort and casecontrol studies. Plast Reconstr Surg 126(6): 2234-2242.

27. Van Dongen SM (2000) Graph clustering by flow simulation. Utrecht University, Amsterdam, NL. pp. 173.

28. Brohee S, Van Helden J (2006) Evaluation of clustering algorithms for protein-protein interaction networks. BMC bioinformatics 7(1): 488 .

29. Cherven K (2015) Mastering Gephi Network Visualization. Packt Publishing, Birmingham, UK. pp. 411.

30. Fan D, Lo CK, Ching V, Kan CW (2014) Occupational health and safety issues in operations management: a systematic and citation network analysis review. Int J Prod Econ 158: 334-344.

31. Newman ME, Girvan M (2004) Finding and evaluating community structure in networks. Phys Rev E 69(2): 026113.

32. Alvarez Nemegyei J, Pelaez Ballestas I, Rodriguez Amado J, Sanin LH, Garcia Garcia C, et al. (2011) Prevalence of rheumatic regional pain syndromes in adults from Mexico: a community survey using COPCORD for screening and syndrome-specific diagnostic criteria. J Rheumatol Suppl 86: 15-20.

33. Alvarez Nemegyei J, Peláez Ballestas I, Goñi M, Flor Julián Santiago, Conrado García-García, et al. (2016) Prevalence of rheumatic regional pain syndromes in Latin-American indigenous groups: a census study based on COPCORD methodology and syndrome-specific diagnostic criteria. Clin Rheumatol 35(1): 63-70.
34. Barrero LH, Pulido JA, Berrio S, Monroy M, Quintana LA, et al. (2012) Physical workloads of the upper extremity among workers of the Colombian flower industry. Am J Ind Med 55(10): 926-939.

35. Leite PC, Merighi MAB, Silva A (2007) The experience of a woman working in nursing suffering from De Quervain's disease. Rev Lat Am Enfermagem 15(2): 253-258.

36. Lin JT, Stubblefield MD (2003) De Quervain's tenosynovitis in patients with lymphedema: a report of 2 cases with management approach. Arch Phys Med Rehabil 84(10): 1554-1557.

37. Milani C, Lin C (2018) Proximallinear extension of skin hypopigmentation after ultrasound-guided corticosteroid injection for de Quervain tenosynovitis: a case presentation. PM\&R 10(8): 873-876.

38. Mardani Kivi M, Mobarakeh MK, Bahrami F, Hashemi Motlagh K, Saheb Ekhtiari K, et al. (2014) Corticosteroid injection with or without thumb spica cast for de Quervain tenosynovitis. J Hand Surg 39(1): 37-41.

39. Mehdinasab SA, Alemohammad SA (2010) Methylprednisolone acetate injection plus casting versus casting alone for the treatment of de Quervain's tenosynovitis. Arch Iran Med 13(4): 270.

40. Pagonis T, Ditsios K, Toli P, Givissis P, Christodoulou A (2011) Improved corticosteroid treatment of recalcitrant de Quervain tenosynovitis with a novel 4-point injection technique. Am J Sports Med 39(2): 398-403.

41. Nayak SR, Krishnamurthy A, Pai MM, Prabhu LV, Ramanathan LA, et al. (2008) Multiple variations of the extensor tendons of the forearm. Rom J Morphol Embryol 49(1): 97-100.

42. Roy S, Mehta V, Suri R, Rath G, Dhuria R, et al. (2010) Bitendinous insertion of abductor pollicis longus coexistent with a rare accessory antebrachial muscle: clinico-anatomical considerations. Clin Ter 161(2): $159-161$.

43. Gurses IA, Coskun O, Gayretli O, Kale A, Ozturk A (2015) The anatomy of the fibrous and osseous components of the first extensor compartment of the wrist: a cadaveric study. Surg Radiol Anat 37(7): 773-777.

44. Schned ES (1986) DeQuervain tenosynovitis in pregnant and postpartum women. Obstet Gynecol 68(3): 411-414.

45. Sit RWS, Tam WH, Chan DCC, Yip BHK, Tam LWY, et al. (2017) A pilot cross-sectional study of postpartum wrist pain in an urban Chinese population: its prevalence and risk factors. Pain physician 20(5): E711-E719.

46. Anderson SE, Steinbach LS, De Monaco D, Bonel HM, Hurtienne Y, et al. (2004) "Baby wrist": MRI of an overuse syndrome in mothers. AJR Am J Roentgenol 182(3): 719-724.

47. Hasan T, Fauzi M (2015) De Quervain's tenosynovitis and phonophoresis: a randomised controlled trial in pregnant females. J Orthop Trauma Rehabil 19(1): 2-6.

48. Turkay R, Inci E, Aydeniz B, Vural M (2017) Shear wave elastography findings of de Quervain tenosynovitis. Eur J Radiol 95: 192-196.

49. Sreejith TG, Althaaf MA (2015) A study on de Quervain's Stenosing Tenosynovitis analyzing the results of different conservative treatment modalities. J Evol Med Dent Sci 4(78): 13649-13654.

50. Rossi C, Cellocco P, Margaritondo E, Bizzarri F, Costanzo G (2005) De Quervain disease in volleyball players. Am J Sports Med 33(3): 424-427. 
ISSN: 2574-1241

DOI: 10.26717/BJSTR.2020.24.004125

Yip Joanne. Biomed J Sci \& Tech Res

(C) This work is licensed under Creative

Submission Link: https://biomedres.us/submit-manuscript.php

$\begin{array}{ll}\text { BIOMEDICAL } & \text { Assets of Publishing with us } \\ \text { RESEARCHES } & \text { - Global archiving of articles } \\ & \text { - Immediate, unrestricted online access } \\ & \text { - Rigorous Peer Review Process } \\ & \text { - Anttps://biomedres.us/ }\end{array}$

